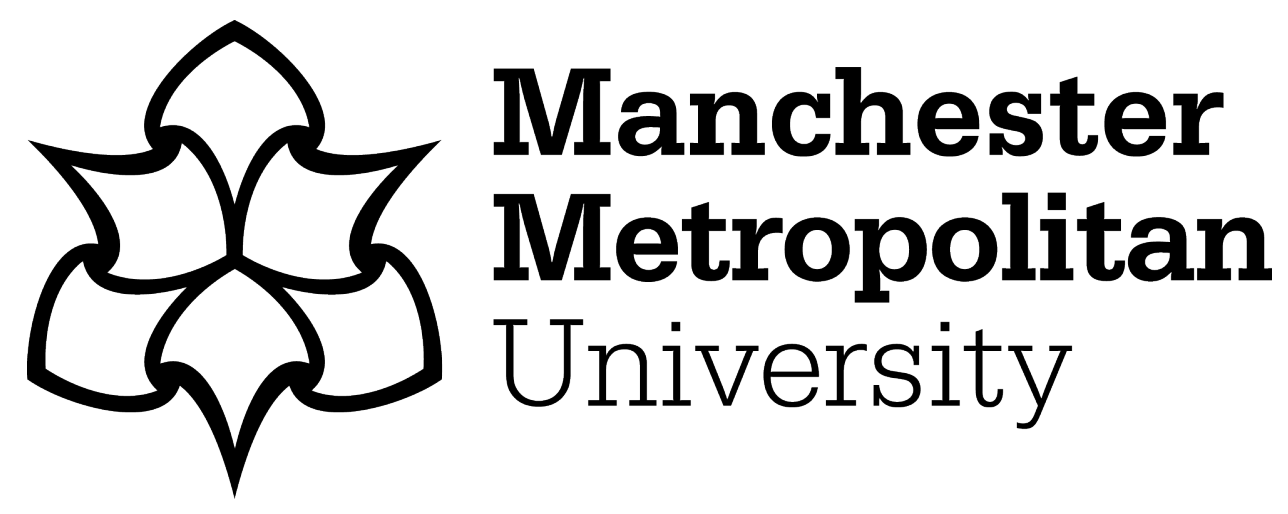

Hogarth, Luke, Burkett, Brendan, van de Vliet, Peter and Payton, Carl ORCID logoORCID: https://orcid.org/0000-0001-8896-9753 (2020) Maximal fully-tethered swim performance in Para swimmers with physical impairment. International Journal of Sports Physiology and Performance, 15 (6). ISSN 1555-0273

Downloaded from: https://e-space.mmu.ac.uk/624727/

Version: Accepted Version

Publisher: Human Kinetics

DOI: https://doi.org/10.1123/ijspp.2019-0515

Please cite the published version 
Maximal fully-tethered swim performance in Para swimmers with physical impairment

Luke Hogarth $^{1}$, Brendan Burkett ${ }^{1}$, Peter Van de Vliet $^{2}$ and Carl Payton ${ }^{3}$

${ }^{1}$ School of Health and Sport Sciences, University of the Sunshine Coast, Australia.

${ }^{2}$ Medical and Scientific Department, International Paralympic Committee, Germany.

${ }^{3}$ Musculoskeletal Science \& Sports Medicine Research Centre, Manchester Metropolitan University, United Kingdom.

Corresponding author:

Luke Hogarth (lhogarth@usc.edu.au)

School of Health and Sport Sciences

University of the Sunshine Coast

Australia

Text-only word count: $\quad 4077$

Abstract word count: $\quad 249$

Number of figures and tables: $\quad 4$

Number of references: $\quad 25$ 


\title{
Maximal fully-tethered swim performance in Para swimmers with physical impairment
}

\begin{abstract}
Purpose: The assessment of swimming propulsion should be a cornerstone of Paralympic swimming classification. However, current methods do not objectively account for this component. This study evaluated the swimming propulsion of swimmers with and without physical impairment using a 30 s maximal fully-tethered freestyle swim test. Methods: Tethered forces were recorded during maximal fully-tethered swimming in eighty competitive swimmers with $(\mathrm{n}=70)$ and without $(\mathrm{n}=10)$ physical impairment. The relationships between absolute and normalised tether forces and maximal freestyle swim speed were established using general additive models. Results: Para swimmers with physical impairment had lower absolute and normalised tether forces than able-bodied swimmers, and there were moderate positive correlations found between tether forces and sport class $(\tau=.52$ to $.55, \mathrm{p}<.001)$. There was a nonlinear relationship between tether force measures and maximal freestyle swim speed in the participant cohort (adj. $R^{2}=.78$ to $\left..80, \mathrm{p}<.001\right)$. Para swimmers with limb deficiency showed stronger relationships between tether force measures and maximal freestyle swim speed (adj. $R^{2}=.78$ to $\left..82, \mathrm{p}<.001\right)$ than for Para swimmers with hypertonia $\left(\operatorname{adj} . R^{2}=.54\right.$ to $\left..73, \mathrm{p}<.001\right)$ and impaired muscle power (adj. $R^{2}=.61$ to $\left..70, \mathrm{p}<.001\right)$. Conclusions: Physical impairments impact on Para swimmers' tether forces during maximal fully-tethered freestyle swimming, explaining a significant proportion of their activity limitation. It is recommended that maximal fully-tethered swimming be included in Paralympic swimming classification as an objective assessment of swimming propulsion.
\end{abstract}

Keywords: Paralympic, propulsion, freestyle, swimming, evidence-based classification. 


\section{Introduction}

In Para swimming a functional classification system has been used to structure competition since the 1992 Barcelona Paralympics. ${ }^{1}$ Swimmers with physical, vision and intellectual impairments compete in separate classes based on the estimated impact of their impairment on swim performance. Swimmers with physical impairment compete across the largest number of classes - ten for freestyle, backstroke and butterfly events (S1-S10) and nine for breaststroke events (SB1-SB9) with lower class numbers indicating greater activity limitation. Eligible types of physical impairment include limb deficiency, leg length difference, short stature, impaired passive range of movement, hypertonia, athetosis, ataxia and impaired muscle power. Swimmers with these eligible types of physical impairments compete in the same classes based on results of dry-land and in-water tests used to estimate their activity limitation in swimming. ${ }^{1}$ The international federation that governs Para swimming has directed research be conducted to guide a revised classification system due to be implemented following the Tokyo 2020 Paralympics. As swimming is conducted in water, the ability to produce propulsion and overcome drag is paramount to performance. ${ }^{2}$ Understanding how eligible impairments affect a Para swimmer's propulsion characteristics should be a cornerstone of the Paralympic swimming classification system. ${ }^{1}$ However, current classification methods do not objectively account for this component.

The measurement of propulsive forces during free swimming is complex due to the aquatic environment. Computational fluid dynamics has been used to estimate many previously immeasurable quantities explaining the forces experienced by the body during swimming. ${ }^{3,4}$ These models require accurate measures of anthropometry and swimming technique, unique computer modelling expertise, and extensive computational power making them unattainable or impractical in many settings. ${ }^{4}$ Maximal tethered swimming is another method that allows for a measurement of propulsive force to be obtained during swimming. This involves attaching an inelastic cord to a swimmer, with the other end attached to a force transducer that is fixed. Force-time data collected during fully-tethered swim trials show good test-retest reliability, ${ }^{5}$ and swimmers have shown muscle activity patterns and physiological responses similar to free swimming of equal duration. ${ }^{6,7}$ Although, altered stroke kinematics occur between fullytethered and free swimming that exaggerate the propulsive force contributions of limb segments. ${ }^{8,9}$ 
Many studies have established the importance of propulsion to swimming performance in ablebodied swimmers using maximal tethered swim tests. These studies have used protocols lasting from $3 \mathrm{~s}$ up to 3 -min to examine the propulsive forces during fully-tethered swimming. The relationship between tether force measures and performance in swim events is influenced by their respective durations, and hence aerobic and anaerobic system contributions. ${ }^{10}$ Tether forces during 3-min maximal tethered swimming are correlated with oxidative metabolism and can be used to evaluate aerobic capacity of swimmers. ${ }^{11,12}$ Tether force variables have limited value in evaluating anaerobic metabolism with this test duration, although mean tether force variables are correlated with performance in short- and middle-distance events. A $30 \mathrm{~s}$ test duration is common within the literature and has been proposed as an adaptation of the Wingate test for swimmer's anaerobic evaluation. ${ }^{6,13}$

The maximum swim velocity a swimmer can attain is limited by the maximum stroking force that they can generate. ${ }^{10}$ This is evidenced by studies that have shown maximum force variables collected during $30 \mathrm{~s}$ all-out tethered swimming to explain the majority of variance in performance in short-distance events. ${ }^{6,14}$ Para swimmers can have physical impairments that affect their ability to generate force needed to overcome drag. ${ }^{15-17}$ For the purpose of classifying these swimmers it is desirable to estimate the reduction in swimming propulsion caused by these limitations rather than the contributions of aerobic and anaerobic capacities that can be increased through effective training. ${ }^{18}$ Measuring the maximum force generating capacities of Para swimmers during short-duration tethered swimming is likely to be the most valid assessment of limitations in swimming propulsion caused by physical impairment.

Currently, there is little information on the maximal fully-tethered swim performance of Para swimmers with physical impairment, and it is unknown how objective propulsive force measurements explain performance in these swimmers. ${ }^{9}$ This study evaluated Para swimmers' propulsion characteristics using a maximal $30 \mathrm{~s}$ fully-tethered swim test. The aims were to: (i) establish differences in tether force measures between swimmers with and without physical impairment, and (ii) establish the relationships between tether force measures, type and severity of physical impairment and freestyle swim performance.

\section{Methods}

Participants 
Eighty competitive swimmers participated in this study; they had limb deficiency $(n=29)$, hypertonia $(n=24)$, impaired muscle power $(n=17)$, or were without physical impairment $(n$ $=10$ ). Participants' characteristics are shown in Table 1. Para swimmers with physical impairment had received international classification with their classifications ranging from S1 to S10. A small group of able-bodied swimmers were included in the study for comparison. All participants were free from injury and undertaking structured training in preparation for competition at the time of testing.

Design

A cross-sectional study design was employed. Participants attended a single test session that involved two components in order; maximal freestyle swimming and maximal fully-tethered swim testing. Maximal freestyle swimming was evaluated over multiple trials using twodimensional video analysis. Force-time data collected during a $30 \mathrm{~s}$ fully-tethered swim test were used to evaluate swimmers' propulsion characteristics.

Methodology

Data were collected in $25 \mathrm{~m}$ and $50 \mathrm{~m}$ swimming pools with a minimum depth of $1.8 \mathrm{~m}$. On arrival, participants' stature and body masses were recorded before they completed their typical activation and pool warm-up. ${ }^{15}$ Warm-up was not standardised due to the large range in impairment severity within the participant cohort. Maximal freestyle swim speed was evaluated through a $10 \mathrm{~m}$ calibrated test zone from video footage using standard two-dimensional video analysis procedures. Two parallel lines $10 \mathrm{~m}$ apart were marked on the pool deck adjacent to the test lane. These lines were extended into the test lane using the line draw tool in video analysis software (Dartfish 7 TeamPro, Dartfish, Fribourg, Switzerland). These lines defined the start and end of the calibrated zone in the pool. The test zone allowed for acceleration and deceleration zones so that participants reached their top speed prior to the test zone and maintained top speed throughout. Video footage from a $50 \mathrm{~Hz}$ video camera (Sony HDRCX700, Sony, Tokyo, Japan) placed in the centre of the test zone and perpendicular to the direction of swimming was captured. The camera was mounted on a tripod in an elevated position and placed on the opposite side of the pool so that distance was a minimum of $15 \mathrm{~m}$ from the plane of motion. Time taken for the participant's first part of the head to contact the start and end lines of the test zone was recorded to the nearest $.02 \mathrm{~s}$ using the video analysis software. Stroke rate (SR), expressed in strokes per minute, was calculated from the number of full stroke cycles (n) completed in the $10 \mathrm{~m}$ test zone and the time (t) taken to perform stroke 
cycles: $\mathrm{SR}=\mathrm{n} / \mathrm{t} \times 60$. Stroke length $(\mathrm{SL})$ was calculated from the swim speed $(\mathrm{v})$ and $\mathrm{SR}: \mathrm{SL}$ $=\mathrm{v} / \mathrm{SR} \times 60$. Participants performed a minimum of two maximal effort trials separated by 3min rest and the fastest trial was used for analysis.

Propulsive force measures were collected using a single all-out $30 \mathrm{~s}$ fully-tethered swim trial that was conducted approximately $10 \mathrm{~min}$ following maximal freestyle swim trials. ${ }^{9} \mathrm{~A}$ submersible in-line load cell (DDEN-500N, Applied Measurements Ltd, Reading, UK) fixed to the pool end wall at $0.5 \mathrm{~m}$ above the water level was used to sample force data at $100 \mathrm{~Hz}$. An inelastic cord linked the load cell to a belt around the participant's waist so that they were situated $5 \mathrm{~m}$ from the pool end wall during tethered swimming. Once participants had familiarised themselves with the tethered swim position, they completed the fully-tethered swim trial using their preferred freestyle swim style. For most swimmers this was front-crawl, although a small number of Para swimmers with impaired muscle power $(\mathrm{n}=4)$ and hypertonia $(n=2)$ used modified swim strokes. Force-time data in the direction of the cable attachment were used to evaluate propulsion characteristics. Data were filtered using a low-pass secondorder Butterworth filter with a $6 \mathrm{~Hz}$ cut-off within SIMI Motion 3D software (SIMI Reality Motion Systems GmBH, version 9.2.0, Unterschleissheim, Germany). Force-time data for trials were divided into six $5 \mathrm{~s}$ windows and the mean tether force was calculated for each window. Several variables were derived, including maximum tether force, average tether force and fatigue index. Maximum tether force expressed in newtons was the highest mean tether force recorded within one of the $5 \mathrm{~s}$ windows. Average tether force expressed in newtons was the mean force recorded over the $30 \mathrm{~s}$ test duration. In addition to absolute values, maximum and average tether force measures were normalised to body mass. Fatigue index was the decline in mean tether force over the $30 \mathrm{~s}$ test. It was calculated from the gradient of mean tether force over the test duration as defined by linear regression and expressed as a percentage of the mean tether force in the first $5 \mathrm{~s}{ }^{19}$

Statistical analysis

Statistics were calculated using R version 3.4.1 (R Core Team, 2017). Normality of distribution and homogeneity of variance of data was confirmed with the Shapiro-Wilk test and Levene's test, respectively. Differences in tether force measures between swimmers with and without physical impairment were determined using a one-way analysis of variance. When a maineffect was found, Bonferroni post hoc tests were conducted and mean differences and 95\% confidence intervals (CI) calculated to determine which types of physical impairment showed 
differences to able-bodied swimmers. The relationships between sport class and tether force measures in Para swimmers were established using Kendall's tau rank correlations, as classes represent ordinal data. A correlation was significant if $p<.05$. Kendall's tau correlations were defined as: weak $<.3$, moderate $.3-.6$, or strong $>.6 .^{20}$

Pearson correlation coefficients were calculated to determine the strength of association between tether force measures and maximal freestyle swim speed. A correlation was significant if $\mathrm{p}<.05$. Pearson's correlations were defined as: weak $<.3$, moderate $.3-.6$, or strong $>.6{ }^{20}$ Generalised additive models were performed to establish the relationship between maximal freestyle swim speed and tether force measures that were correlated. Coefficient of determination $\left(R^{2}\right)$ statistics were calculated to determine the amount of variance in maximal freestyle swim speed explained by generalised additive models. In addition to combined analysis, Para swimmers with limb deficiency, hypertonia or impaired muscle power were analysed independently to examine the influence of type of physical impairment on the relationship between tether force measures and maximal freestyle swim speed.

\section{Results}

The maximal freestyle swim speeds ranged between $0.21-1.79 \mathrm{~m} \cdot \mathrm{s}^{-1}$ in Para swimmers with physical impairment and between $1.58-1.93 \mathrm{~m} \cdot \mathrm{s}^{-1}$ in able-bodied swimmers. There was a main effect of type of physical impairment on maximal freestyle swim speed $\left(\mathrm{F}_{3,75}=12.4, \mathrm{p}<.001\right)$ and stroke length $\left(\mathrm{F}_{3,75}=9.6, \mathrm{p}<.001\right)$. Bonferroni post hoc tests showed Para swimmers had lower swim speeds (limb deficiency: $-0.4[-0.26,-0.54] \mathrm{m} \cdot \mathrm{s}^{-1}, \mathrm{p}=.006$; hypertonia: -0.58 [$0.41,-0.76] \mathrm{m} \cdot \mathrm{s}^{-1}, \mathrm{p}<.001$; impaired muscle power: $\left.-0.84[-.65,-1.04] \mathrm{m} \cdot \mathrm{s}^{-1}, \mathrm{p}<.001\right)$ and stroke lengths (limb deficiency: $-0.44[-0.26,-0.62] \mathrm{m}, \mathrm{p}=.01$; hypertonia: -0.54 [-0.33, -0.75$]$ $\mathrm{m}, \mathrm{p}=.001$; impaired muscle power: $-0.75[-0.52,-0.99] \mathrm{m}, \mathrm{p}<.001)$ than able-bodied swimmers (Figure 1A and 1E). There were moderate to strong, positive correlations found between Para swimmers' swim speeds $(\tau=.63, \mathrm{p}<.001)$ and stroke lengths $(\tau=.51, \mathrm{p}<.001)$ with their sport class (Figures $1 \mathrm{~B}$ and $1 \mathrm{~F}$ ). There was no main effect of type of physical impairment found on stroke rate $\left(\mathrm{F}_{3,75}=2.5, \mathrm{p}=.08\right)$. Although, there were greater variances in stroke rates for Para swimmers than for able-bodied swimmers (Figure 1C), and there was a weak positive correlation between stroke rate and sport class $(\tau=.29, \mathrm{p}=.001)$.

Absolute maximum and average tether forces ranged between 8.1-204.0 N and 6.5-188.6 $\mathrm{N}$ in the participant cohort equating to values normalised to body mass of $0.14-2.34 \mathrm{~N} \cdot \mathrm{kg}^{-1}$ and 0.11 - 
2.16 $\mathrm{N} \cdot \mathrm{kg}^{-1}$, respectively. Fully-tethered swim measures for participants stratified by type of physical impairment and sport class are shown in Figure 2. There was a main effect of type of physical impairment on absolute maximum tether force $\left(\mathrm{F}_{3,76}=12.9, \mathrm{p}<.001\right)$, normalised maximum tether force $\left(F_{3,76}=10.8, p<.001\right)$, absolute average tether force $\left(F_{3,76}=14.1, p\right.$ $<.001)$ and normalised average tether force $\left(\mathrm{F}_{3,76}=11.7, \mathrm{p}<.001\right)$. Para swimmers with impaired muscle power and hypertonia showed the largest differences in absolute maximum tether force (hypertonia: $-54.8[-26.7,-83.1] \mathrm{N}, \mathrm{p}<.001$; impaired muscle power: -73.7 [-45.9, $-101.6] \mathrm{N}, \mathrm{p}<.001$ ), normalised maximum tether force (hypertonia: $-0.76[-0.45,-1.06] \mathrm{N} \cdot \mathrm{kg}^{-}$ $1, \mathrm{p}<.001$; impaired muscle power: $\left.-0.88[-0.57,-1.20] \mathrm{N} \cdot \mathrm{kg}^{-1}, \mathrm{p}<.001\right)$, absolute average tether force (hypertonia: $-50.1[-23.7,-76.4] \mathrm{N}, \mathrm{p}<.001$; impaired muscle power: -63.9 [-37.6, $-90.2] \mathrm{N}, \mathrm{p}<.001$ ), and normalised average tether force (hypertonia: $-0.67[-0.39,-0.85] \mathrm{N} \cdot \mathrm{kg}^{-}$ $1, \mathrm{p}<.001$; impaired muscle power: $\left.-0.75[-0.46,-1.04] \mathrm{N} \cdot \mathrm{kg}^{-1}, \mathrm{p}<.001\right)$ compared to ablebodied swimmers. Although differences were smaller, Para swimmers with limb deficiency also showed lower maximum tether forces $(-46.8[-19.0,-74.7] \mathrm{N}, \mathrm{p}<.001 ;-0.47[-0.16,-0.78]$ $\left.\mathrm{N} \cdot \mathrm{kg}^{-1}, \mathrm{p}<.001\right)$ and average tether forces $(-41.8[-15.6,-68.0] \mathrm{N}, \mathrm{p}<.001 ;-0.41[-0.12,-0.70]$ $\left.\mathrm{N} \cdot \mathrm{kg}^{-1}, \mathrm{p}=.02\right)$ than able-bodied swimmers. There was no main effect of type of physical impairment on fatigue index $\left(\mathrm{F}_{3,76}=0.04, \mathrm{p}=.98\right)$. There were moderate positive correlations between absolute and normalised maximum tether forces and sport class in Para swimmers $(\tau$ $=.55, \mathrm{p}<.001)$. Similar correlations were found between absolute and normalised average tether forces and sport class $(\tau=.54, \mathrm{p}<.001)$. Fatigue index had a weak positive correlation with sport class $(\tau=.23, \mathrm{p}=.01)$.

The was a nonlinear relationship between absolute and normalised tether force variables and maximal freestyle swim speed (Figure 3). Generalised additive models showed tether force measures to independently explain between $75.9 \%$ and $80.7 \%$ of the deviance in maximal freestyle swim speed in the participant cohort, with absolute tether forces (adj. $R^{2}=.78$ to .80 , $\mathrm{p}<.001)$ reporting slightly higher coefficients of determination than tether forces normalised to body mass (adj. $R^{2}=.75, \mathrm{p}<.001$ ). The relationships between tether force variables and maximal freestyle swim speed in Para swimmers with different types of physical impairment are shown in Figure 3. Generalised additive models explained the most variance in freestyle swim performance from tether force measures in Para swimmers with limb deficiency (adj. $R^{2}$ $=.78$ to $.82, \mathrm{p}<.001)$. There were lower coefficients of determination reported for generalised additive models in Para swimmers with impaired muscle power $\left(\operatorname{adj} . R^{2}=.61\right.$ to $\left..70, \mathrm{p}<0.01\right)$ and Para swimmers with hypertonia (adj. $R^{2}=.54$ to $.73, \mathrm{p}<.001$ ). Para swimmers with 
impaired muscle power showed stronger relationships between maximal freestyle swim performance and tether force variables that were normalised to body mass (adj. $R^{2}=.66$ to .70 , $\mathrm{p}<.001$ ), rather than absolute values (adj. $R^{2}=.61$ to $.63, \mathrm{p}<.001$ ). The opposite was found for Para swimmers with hypertonia (Figure 3).

\section{Discussion}

The objective assessment of swimming propulsion and how it is affected by swimmers' physical impairments should be a cornerstone of Paralympic swimming classification. This study used a maximal fully-tethered freestyle swim test to quantify the propulsive force characteristics of swimmers with and without physical impairment. It was found that absolute and normalised tether force measures differed between swimmers with and without physical impairment and decreased with greater severity of impairment in Para swimmers as indicated by their sport class. Tether force measures were strongly correlated with maximal freestyle swim performance, although there appeared to be stronger relationships found for Para swimmers with limb deficiency than for other types of physical impairment. These results support the inclusion of the maximal fully-tethered swim test in Paralympic swimming classification to provide an objective assessment of swimming propulsion.

The average and maximum tether forces reported for able-bodied swimmers in this study ranged between 76.8-188.6 N (mean 109.8 $\pm 35.1 \mathrm{~N}$ ) and 89.4-204.0 N (mean 127.2 $\pm 36.7 \mathrm{~N}$ ), respectively. These values sit within or above the range of tether forces published in ablebodied swimmers using the same test duration, for example, Lee et al. ${ }^{9}$, female swimmers (n= 9) average force $71.0 \pm 8.9 \mathrm{~N}$ and maximum force $80.8 \pm 10.6 \mathrm{~N}$; Morouco et al. ${ }^{14}$, male swimmers $(\mathrm{n}=12)$ average force $98.8 \pm 13.7 \mathrm{~N}$, female swimmers $(\mathrm{n}=11)$ average force 74.0 $\pm 12.4 \mathrm{~N}$; and Morouco et al. ${ }^{6}$, male swimmers $(\mathrm{n}=34)$ average force $112.7 \pm 15.6 \mathrm{~N}$. This suggests the tether force values reported for able-bodied swimmers in this study provide an appropriate benchmark to compare Para swimmers' performances despite the small sample.

Para swimmers had lower absolute and normalised tether forces than able-bodied swimmers, confirming that their physical impairments impact on swimming propulsion. This was true even in those Para swimmers from the higher sport classes $(n=19, \geq S 9)$ that have the least severe impairments. These swimmers had lower average $(79.9 \pm 20.5 \mathrm{~N}$ vs. $109.8 \pm 35.1 \mathrm{~N}, \mathrm{p}=.03)$ and maximum $(95.3 \pm 25.3 \mathrm{~N}$ vs. $127.2 \pm 36.7 \mathrm{~N}, \mathrm{p}=.03)$ tether forces than able-bodied 
swimmers. Previous research has shown Para swimmers with a single, through elbow limb deficiency $(\mathrm{n}=9, \mathrm{~S} 9)$ to produce an average tether force of $55.7 \pm 3.5 \mathrm{~N}$, equating to approximately $20 \%$ less net propulsive force than able-bodied swimmers with comparable training backgrounds. ${ }^{9}$ The current study together with the study by Lee et al. ${ }^{9}$ shows that Para swimmers with physical impairment, even those with the least severe impairments, produce lower net propulsive force during maximal fully tethered swimming than able-bodied swimmers.

The tether forces reported for Para swimmers were associated with the degree of their swimspecific impairment as defined by the current classification system (Figure 2). There were moderate positive correlations between average and maximum tether forces and sport class $(\tau$ $=.54$ to $.55, \mathrm{p}<.001)$, showing that Para swimmers create less propulsive force when swimming as severity of impairment increases. Similar observations were shown for maximal freestyle swim speed (Figure 1B) and stroke length (Figure 1F). This highlights the potential of using tether force measures to gain an objective assessment of Para swimmers' activity limitation to help guide their classification. However, the stronger correlation found between maximal freestyle swim speed and sport class $(\tau=.63, \mathrm{p}<.001)$ highlights the fact that Para swimmers can also have higher active and passive drag than able-bodied swimmers that explains a separate proportion of activity limitation in Para swimming. ${ }^{20}$

The fatigue index during fully-tethered swimming, describing the decline in tether force over the test duration, did not differ between swimmers with and without physical impairment (Figure 2I). The fatigue indexes reported for able-bodied swimmers in this study $(30 \pm 8 \%)$ were between the values of $22 \pm 7 \%$ reported by Lee et al. ${ }^{9}$ and $38 \pm 8 \%$ reported by Morouco et al. ${ }^{13}$. Para swimmers with limb deficiency $(29 \pm 12 \%)$, hypertonia $(30 \pm 12 \%)$, and impaired muscle power $(29 \pm 14 \%)$ showed similar mean values to able-bodied swimmers, suggesting that the ability to maintain propulsive forces during short, maximal freestyle swimming is not influenced by type of physical impairment. This refutes anecdotal evidence that Para swimmers with hypertonia, including medical conditions like cerebral palsy and acquired brain injury, are at an increased disadvantage due to "tying up" towards the end of an event. This phenomenon has been evidenced in Para athletes with cerebral palsy that show neuromuscular irregularities towards the end of maximal exercise performance, including bilateral coactivation, atypical firing patterns and continuous irregular muscle activation ${ }^{21}$ that negatively affects performance through the selection of a conservative pacing strategy. ${ }^{22}$ The similar fatigue indexes for 
swimmers with different types of physical impairment in this study (Figure 2I) suggests that classification should not account for this phenomenon, at least in the short-distance events, until further research defines the determining factors influencing pacing and fatigue profiles in these athletes during maximal swimming.

Tether force variables independently explained the majority of variance in maximal freestyle swim speed in this study's participant cohort (adj. $R^{2}=.75$ to $\left..80, \mathrm{p}<.001\right)$. There was a nonlinear relationship found between average and maximum tether force measures and maximal swim speed, showing that the capability to generate net propulsive forces to overcome drag becomes increasingly important with slower maximal swim speeds (Figure 3). This suggests that smaller absolute differences in tether force values between Para swimmers in the lower sport classes can have a stronger influence on activity limitation, perhaps as these swimmers also have impairments causing higher drag than swimmers in higher sport classes. ${ }^{20}$

The relationship between tether force measures and maximal freestyle swim speed appeared to be influenced by type of physical impairment (Figure 3). Tether force measures explained the majority of variance in maximal freestyle swim speed in Para swimmers with limb deficiency (adj. $R^{2}=.78$ to $\left..82, \mathrm{p}<.001\right)$. It appears these swimmers have reduced limb length and surface area that predominately impacts their ability to generate propulsive forces during swimming, rather than causing increased active or passive drag. ${ }^{23}$ Comparatively, there were lower coefficients of determination reported for Para swimmers with impaired muscle power (adj. $R^{2}$ $=.63$ to $.70, \mathrm{p}<.001)$ and hypertonia (adj. $R^{2}=.54$ to $\left..73, \mathrm{p}<.001\right)$. For these swimmers, it is possible that impairments in motor coordination or range of movement predispose them to increased form drag or limits their ability to minimise the disturbance of the water during the swim stroke. ${ }^{24,25}$ The combination of objective measures of propulsion and drag may better explain the variance in swim performance for Para swimmers with hypertonia and impaired muscle power.

Although this study has important implications for Para swimming classification there are several limitations that should be addressed. This study reports the propulsion characteristics in the largest sample of Para swimmers to date, yet further research is required to establish normative values in a larger sample of Para swimmers stratified by sex, age, and type and severity of physical impairment. It is also important to consider the influence of training status on the relationship between type and severity of physical impairment and fully-tethered swim performance in Para swimmers. Classification should consider the mode, frequency and 
volume of Para swimmers' training given that fully-tethered swim performance is likely to be highly training dependent. This study can guide further research efforts to better understand the influence of sex, age, type and severity of impairment, and training status on propulsion characteristics in Para swimmers with physical impairment.

\section{Practical applications}

It is recommended that the maximal fully-tethered swim test be included in Paralympic swimming classification as an objective assessment of swimming propulsion. The normative data in this study may be used to benchmark performances of Para swimmers to guide their classification. A larger sample of able-bodied swimmers stratified by sex and training status will allow for these factors to be accounted for during athlete benchmarking. These results also have implications for testing and training in Para swimmers with physical impairment. Tether force measures during the maximal fully-tethered swim test were found to be important determinants of freestyle swim performance in these swimmers. Improving the capacity to generate swimming propulsion should be a key objective of their training, and the maximal fully-tethered swim test can be used to monitor the development and maintenance of swimming propulsion.

\section{Conclusions}

This study examined the swimming propulsion characteristics of Para swimmers with and without physical impairment using a maximal $30 \mathrm{~s}$ fully-tethered swim test. Tether force measures were lower in Para swimmers with physical impairment than for able-bodied swimmers, decreased with greater severity of swimming-specific impairment as defined by the current classification system, and explained the majority of variance in freestyle swim performance. These results support the inclusion of the maximal fully-tethered swim test in Paralympic swimming classification to provide an objective assessment of the impact that Para swimmers' physical impairments have on swimming propulsion. Importantly, the type of physical impairment influences the relationship between tether force measures and freestyle swimming performance. Further research is required to examine the relative contribution of propulsion and drag measures to swim performance, particularly in Para swimmers with 
hypertonia and impaired muscle power for whom drag measures may be more important in explaining activity limitation.

\section{Acknowledgements}

This research was supported by the International Paralympic Committee and UK Sport. 


\section{References}

1. Burkett B, Payton C, Van de Vliet P, et al. Performance characteristics of Para swimmers: How effective is the swimming classification system? Phys Med Rehabil Clin N Am. 2018;29(2):333-346.

2. Toussaint HM, Beek PJ. Biomechanics of competitive front crawl swimming. Sports Med. 1992;13(1):8-24.

3. Cohen RCZ, Cleary PW, Mason BR, Pease DL. Forces during front crawl swimming at different stroke rates. Sports Eng. 2017;21(1):63-73.

4. Takagi H, Nakashima M, Sato Y, Matsuuchi K, Sanders RH. Numerical and experimental investigations of human swimming motions. J Sport Sci. 2016;34(16):1564-1580.

5. Kjendlie J, Thorsvald K. A tethered swimming test is highly reliable. Portuguese Journal of Sport Sciences. 2006;6:231-233.

6. Morouco PG, Marinho DA, Keskinen KL, Badillo JJ, Marques MC. Tethered Swimming Can Be Used to Evaluate Force Contribution for Short-Distance Swimming Performance. J Strength Cond Res. 2014;28(11):3093-3099.

7. Bollens E, Annemans L, Vaes W, Clarys J. Peripheral EMG comparison between fully tethered and free front crawl swimming. In: Ungerechts B, Wilke K, Reischle K, eds. Swimming Science V. Champaign, IL: Human Kinetics; 1988:173-181.

8. Samson M, Monnet T, Bernard A, Lacouture P, David L. Comparative study between fully tethered and free swimming at different paces of swimming in front crawl. Sports Biomech. 2018:1-16.

9. Lee CJ, Sanders RH, Payton CJ. Changes in force production and stroke parameters of trained able-bodied and unilateral arm-amputee female swimmers during a $30 \mathrm{~s}$ tethered front-crawl swim. J Sports Sci. 2014;32(18):1704-1711.

10. Amaro NM, Morouco PG, Marques MC, Fernandes RJ, Marinho DA. Biomechanical and bioenergetical evaluation of swimmers using fully-tethered swimming: A qualitative review. J Hum Sport Exerc. 2017;12(4):1346-1360.

11. Kalva CA, Zagatto AM, da Silva AS, de Araujo MYC, de Almeida PB, Papoti M. Tethered 3-min all-out test did not predict the traditional critical force parameters in inexperienced swimmers. J Sport Med Phys Fit. 2017;57(9):1126-1131.

12. Kalva CA, Zagatto AM, da Silva ASR, et al. Relationships among the tethered 3-min allout test, MAOD and swimming performance. Int J Sports Med. 2017;38(5):353-358. 
13. Morouco P, Keskinen KL, Vilas-Boas JP, Fernandes RJ. Relationship between tethered forces and the four swimming techniques performance. J Appl Biomech. 2011;27(2):161169.

14. Morouco PG, Marinho DA, Izquierdo M, Neiva H, Marques MC. Relative contribution of arms and legs in $30 \mathrm{~s}$ fully tethered front crawl swimming. Biomed Res Int. 2015;2015:563206.

15. Hogarth L, Nicholson V, Spathis J, et al. A battery of strength tests for evidence-based classification in Para swimming. J Sports Sci. 2018;37(4):1-10.

16. Dingley AA, Pyne D, Burkett B. Dry-land bilateral hand-force production and swimming performance in paralympic swimmers. Int J Sports Med. 2014;35(11):949-953.

17. Morouco P, Neiva H, Gonzalez-Badillo JJ, Garrido N, Marinho DA, Marques MC. Associations between dry land strength and power measurements with swimming performance in elite athletes: a pilot study. J Hum Kinet. 2011;29A:105-112.

18. Tweedy SM, Vanlandewijck YC. International Paralympic Committee position stand background and scientific principles of classification in Paralympic sport. Brit J Sport Med. 2011;45(4):259-269.

19. Morouco PG, Vilas-Boas JP, Fernandes RJ. Evaluation of adolescent swimmers through a 30-s tethered test. Pediatr Exerc Sci. 2012;24(2):312-321.

20. Oh YT, Burkett B, Osborough C, Formosa D, Payton C. London 2012 Paralympic swimming: passive drag and the classification system. Brit J Sport Med. 2013;47(13):838843.

21. Runciman P, Derman W, Ferreira S, Albertus-Kajee Y, Tucker R. A descriptive comparison of sprint cycling performance and neuromuscular characteristics in ablebodied athletes and paralympic athletes with cerebral palsy. Am J Phys Med Rehab. 2015;94(1):28-37.

22. Runciman P, Tucker R, Ferreira S, Albertus-Kajee Y, Derman W. Paralympic athletes with cerebral palsy display altered pacing strategies in distance-deceived shuttle running trials. Scand J Med Sci Sports. 2016;26(10):1239-1248.

23. Hogarth L, Payton C, Van de Vliet P, Connick M, Burkett B. A novel method to guide classification of para swimmers with limb deficiency. Scand J Med Sci Sports. 2018;28(11):2397-2406.

24. Hogarth L, Payton C, Nicholson V, et al. Classifying motor coordination impairment in Para swimmers with brain injury. J Sci Med Sport. 2019;22(5):526-531. 
25. Nicholson VP, Spathis JG, Hogarth LW, et al. Establishing the reliability of a novel battery of range of motion tests to enable evidence-based classification in Para Swimming. Phys Ther Sport. 2018;32:34-41. 


\section{Figure captions}

Figure 1. Maximal freestyle swim performance and stroke parameters in swimmers stratified by type of physical impairment and sport class. Sport classes range from S1 to S10 with lower class numbers indicating greater activity limitation than higher class numbers in the freestyle, backstroke and butterfly swim events. Male (grey) and female (white) swimmers are identified by the shade of data points.

Figure 2. Tether force measures during maximal fully-tethered freestyle swimming in swimmers stratified by type of physical impairment and sport class. Sport classes range from $\mathrm{S} 1$ to S10 with lower class numbers indicating greater activity limitation than higher class numbers in the freestyle, backstroke and butterfly swim events. Male (grey) and female (white) swimmers are identified by the shade of data points.

Figure 3. Relationships between tether force measures and maximal freestyle swim speed in swimmers with physical impairment. Male (grey) and female (white) swimmers are identified by the shade of data points. The Smooth line shows the relationship in the entire participant cohort (transparent data points) fitted with general additive models. Coefficients of determination $\left(R^{2}\right)$ and correlations $(r)$ show the relationships between variables in Para swimmers with physical impairment as determined by separate general additive models. ** Indicates $p<.001$ 

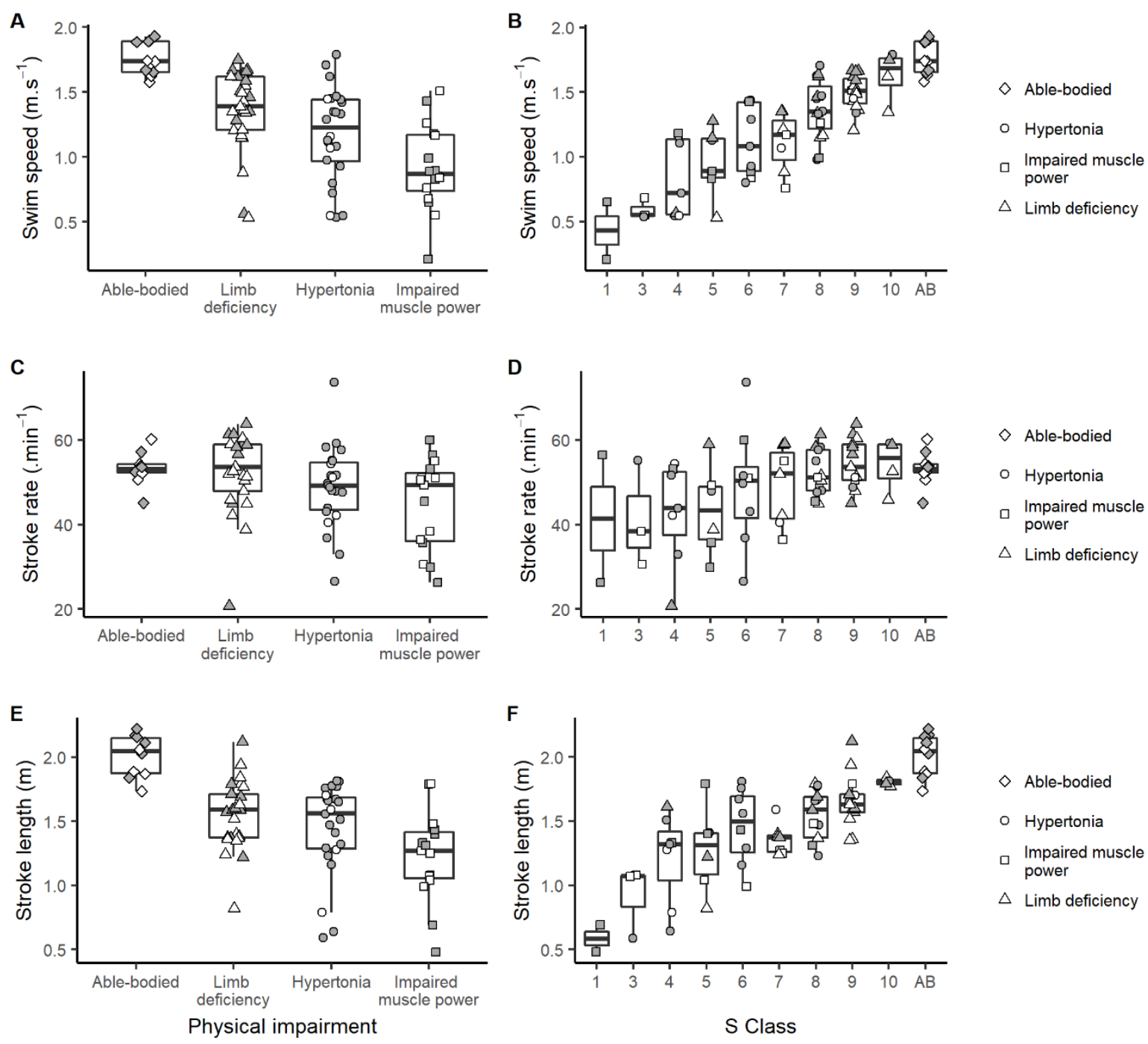

Figure 1. Maximal freestyle swim performance and stroke parameters in swimmers stratified by type of physical impairment and sport class. Sport classes range from S1 to S10 with lower class numbers indicating greater activity limitation than higher class numbers in the freestyle, backstroke and butterfly swim events. Male (grey) and female (white) swimmers are identified by the shade of data points. 

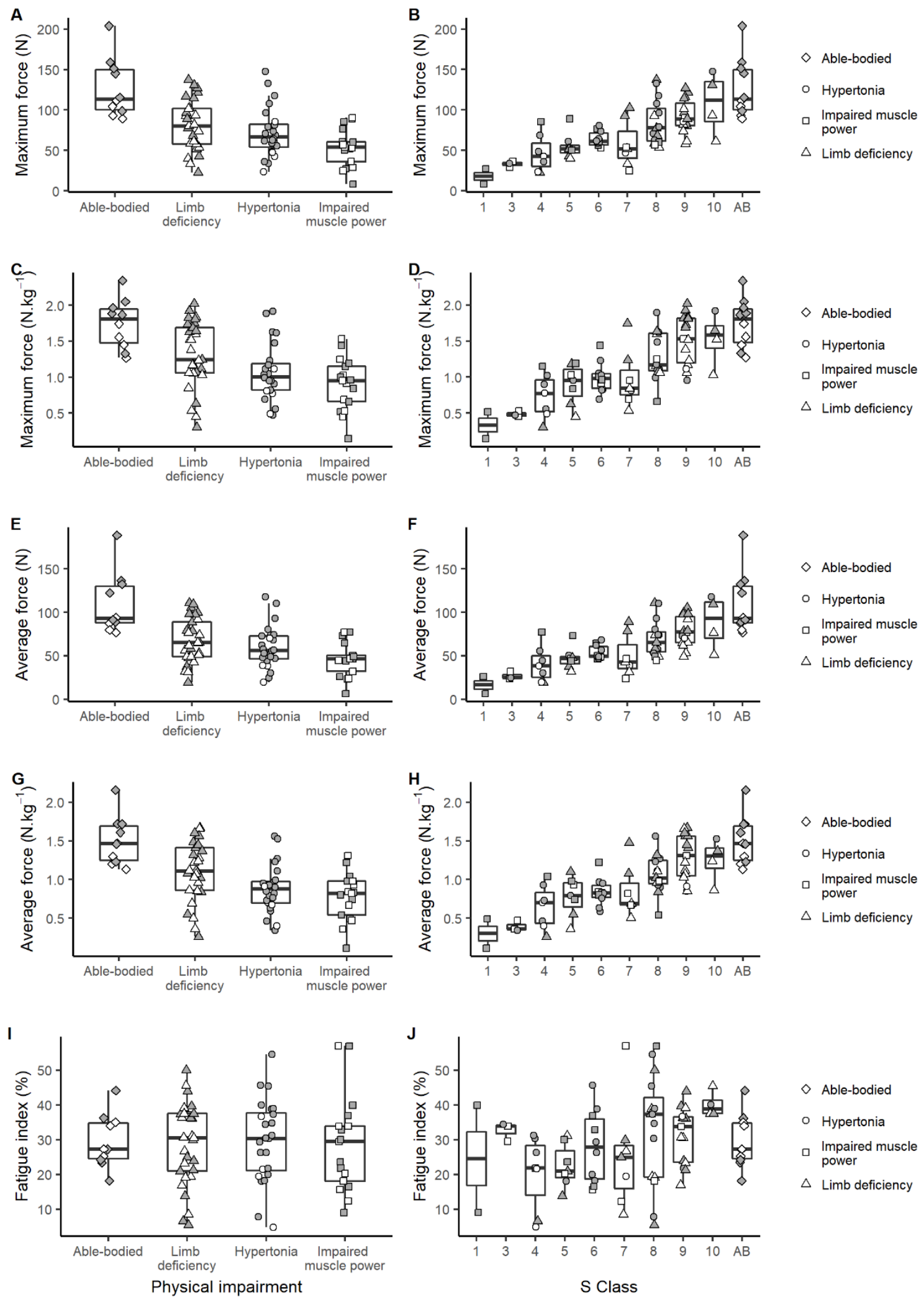

Figure 2. Tether force measures during maximal fully-tethered freestyle swimming in swimmers stratified by type of physical impairment and sport class. Sport classes range from $\mathrm{S} 1$ to S10 with lower class numbers indicating greater activity limitation than higher class numbers in the freestyle, backstroke and butterfly swim events. Male (grey) and female (white) swimmers are identified by the shade of data points. 

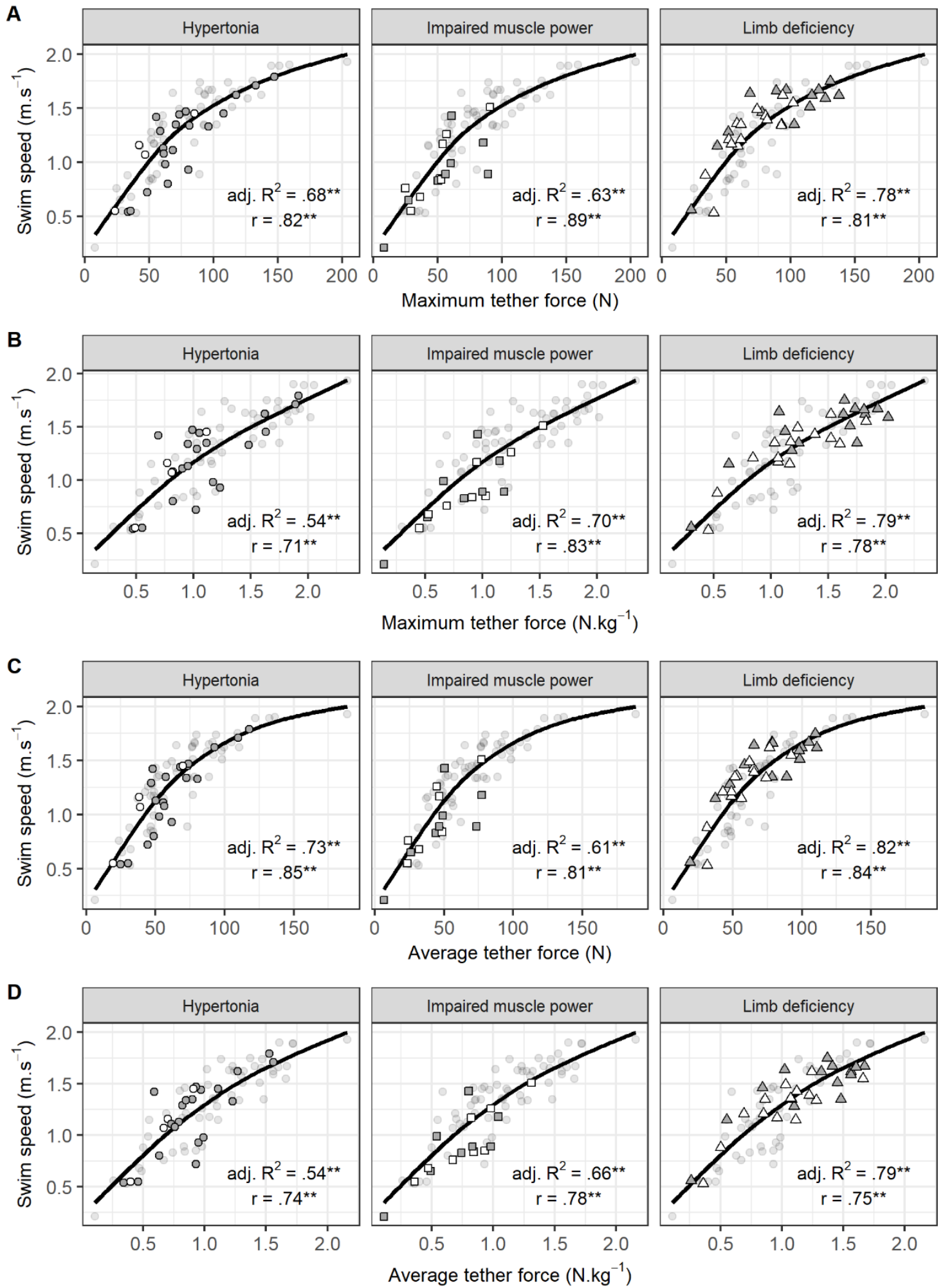

Figure 3. Relationships between tether force measures and maximal freestyle swim speed in swimmers with physical impairment. Male (grey) and female (white) swimmers are identified by the shade of data points. The Smooth line shows the relationship in the entire participant cohort (transparent data points) fitted with general additive models. Coefficients of determination $\left(R^{2}\right)$ and correlations $(r)$ show the relationships between variables in Para swimmers with physical impairment as determined by separate general additive models. ** Indicates $\mathrm{p}<.001$ 


\section{Tables}

Table 1. Characteristics of able-bodied swimmers and Para swimmers with physical impairments.

\begin{tabular}{|c|c|c|c|c|c|}
\hline & & Limb deficiency & Hypertonia & $\begin{array}{c}\text { Impaired muscle } \\
\text { power }\end{array}$ & Able-bodied \\
\hline & Males & $n=15$ & $n=20$ & $n=9$ & $n=6$ \\
\hline & Females & $n=14$ & $n=4$ & $n=8$ & $n=4$ \\
\hline \multirow[t]{2}{*}{ Age (years) } & Males & $20.3(4.2)$ & $25.3(6.4)$ & $34.8(5.3)$ & $21.5(4.5)$ \\
\hline & Females & $21.3(5.2)$ & $21.5(7.5)$ & $30.6(11.7)$ & $19.3(2.4)$ \\
\hline \multirow[t]{2}{*}{$\begin{array}{l}\text { Body mass } \\
(\mathrm{kg})\end{array}$} & Males & $65.4(11.9)$ & $69.3(9.5)$ & $64.8(12.7)$ & $76.0(9.6)$ \\
\hline & Females & $59.2(9.6)$ & $59.8(12.3)$ & $54.8(10.7)$ & $66.4(3.0)$ \\
\hline \multirow[t]{2}{*}{ Stature $(\mathrm{cm})$} & Males & $170.0(21.5)$ & $172.7(8.9)$ & $169.6(10.9)$ & $178.9(10.5)$ \\
\hline & Females & $161.6(15.2)$ & $158.2(11.8)$ & $152.1(14.8)$ & $172.7(7.0)$ \\
\hline \multirow[t]{9}{*}{ S Class } & & $\mathrm{S} 1(n=0)$ & $\mathrm{S} 1(n=0)$ & $\mathrm{S} 1(n=2)$ & \\
\hline & & $\mathrm{S} 3(n=0)$ & $\mathrm{S} 3(n=1)$ & $\mathrm{S} 3(n=2)$ & \\
\hline & & $\mathrm{S} 4(n=1)$ & $\mathrm{S} 4(n=5)$ & $\mathrm{S} 4(n=1)$ & \\
\hline & & $\mathrm{S} 5(n=3)$ & $\mathrm{S} 5(n=1)$ & $\mathrm{S} 5(n=3)$ & \\
\hline & & $\mathrm{S} 6(n=0)$ & $\mathrm{S} 6(n=6)$ & $\mathrm{S} 6(n=4)$ & \\
\hline & & $\mathrm{S} 7(n=4)$ & $\mathrm{S} 7(n=1)$ & $\mathrm{S} 7(n=2)$ & \\
\hline & & $\mathrm{S} 8(n=6)$ & $\mathrm{S} 8(n=7)$ & $\mathrm{S} 8(n=2)$ & \\
\hline & & $\mathrm{S} 9(n=12)$ & $\mathrm{S} 9(n=2)$ & $\mathrm{S} 9(n=1)$ & \\
\hline & & $\mathrm{S} 10(n=3)$ & $\mathrm{S} 10(n=1)$ & $\mathrm{S} 10(n=0)$ & \\
\hline \multirow[t]{2}{*}{ Standard $^{\mathrm{a}}$} & & $\begin{array}{c}\text { International }(\mathrm{n}= \\
12)\end{array}$ & $\begin{array}{l}\text { International (n } \\
\quad=13)\end{array}$ & $\begin{array}{l}\text { International (n } \\
=9)\end{array}$ & $\begin{array}{c}\text { International (n } \\
=4)\end{array}$ \\
\hline & & National $(\mathrm{n}=17)$ & $\begin{array}{c}\text { National }(\mathrm{n}= \\
11)\end{array}$ & National $(\mathrm{n}=8)$ & National $(\mathrm{n}=6)$ \\
\hline \multirow[t]{4}{*}{$\begin{array}{l}\text { Medical } \\
\text { conditions }\end{array}$} & & Acquired $(\mathrm{n}=8)$ & $\begin{array}{l}\text { Acquired brain } \\
\text { injury }(n=2)\end{array}$ & $\begin{array}{l}\text { Charcot-Marie } \\
\text { Tooth disease (n } \\
=3 \text { ) }\end{array}$ & \\
\hline & & $\begin{array}{c}\text { Congenital }(\mathrm{n}= \\
21)\end{array}$ & $\begin{array}{c}\text { Diplegic CP }(\mathrm{n}= \\
8)\end{array}$ & $\begin{array}{c}\text { Complete SCI (n } \\
=7)\end{array}$ & \\
\hline & & & $\begin{array}{c}\text { Hemiplegic CP } \\
(\mathrm{n}=7)\end{array}$ & $\begin{array}{l}\text { Incomplete SCI } \\
(\mathrm{n}=5)\end{array}$ & \\
\hline & & & $\begin{array}{c}\text { Hereditary } \\
\text { spastic } \\
\text { quadriplegia }(\mathrm{n}= \\
1) \\
\text { Quadriplegic CP } \\
(\mathrm{n}=6)\end{array}$ & $\begin{array}{c}\text { Spina bifida }(\mathrm{n}= \\
2)\end{array}$ & \\
\hline
\end{tabular}

CP: Cerebral palsy, SCI: Spinal cord injury. ${ }^{\text {a }}$ Participants were classified as international standard if they had been selected to represent their nation at a Paralympic, Olympic, World Championship or Commonwealth games event otherwise they were classified as national standard. 